\title{
Evaluation du niveau de transfert de métaux lourds (cadmium, cuivre, plomb et zinc) dans Lactuca sativa $L$. co-cultivée avec Cymbopogon citratus (DC) Stapf
}

\author{
Issaka SENOU $1,2^{\star}$, Mamadou NIMI ${ }^{3}$, Hassan B. NACRO4, Antoine N. SOME1 \\ ${ }^{1}$ Laboratoire des Systèmes Naturels, des Agrosystèmes et de l'Ingénierie de l'Environnement (Sy.N.A.I.E), Institut du \\ Développement Rural (I.D.R), Université Nazi BONI (U.N.B) Bobo-Dioulasso, BP 1091, Bobo-Dioulasso, Burkina \\ Faso \\ 2Institut des Sciences de l'Environnement et du Développement Rural, Université de Dédougou (UDDG), BP : 176, \\ Dédougou, Burkina Faso \\ ${ }^{3}$ Laboratoire de géochimie du Bureau des Mines et de la Géologie du Burkina (BUMIGEB), Bobo Bobo-Dioulasso, \\ Burkina Faso \\ ${ }^{4}$ Laboratoire d'étude et de recherche sur la fertilité du sol (L.E.R.F), Institut du Développement Rural (I.D.R), \\ Université Nazi BONI (U.N.B); BP 1091, Bobo-Dioulasso, Burkina Faso \\ *Auteur pour correspondance : issakasenou@gmail.com
}

Original submitted in on $4^{\text {th }}$ September 2019. Published online at www.m.elewa.org/journals/ on 31st December 2019 https://doi.org/10.35759/JABs.v144.8

\section{RESUME}

Objectifs : Evaluer les effets des associations laitue et citronnelle (Cymbopogon citratus (DC) Stapf) sur le transfert de métaux lourds dans les organes de la laitue (Lactuca sativa L.)

Méthodologie et résultats : Un essai en plein champ a été conduit. Le dispositif expérimental était en blocs factoriels complètement randomisés comprenant trois traitements et trois répétitions. Les traitements ont comparé la culture pure de la laitue (T0) aux associations laitue-citronnelle alternés sur la même ligne (T1) et laitue-citronnelle où les plants de citronnelle sont placés dans les interlignes de la laitue (T2). Les analyses ont été faites sur un Spectromètre à Absorption Atomique (SAA) à flamme. L'appareil utilisé est un Perkin Helmer AAnalyst100. Le dispositif était géré par un ordinateur. Pour chaque élément, la valeur de la concentration affichée par l'ordinateur est obtenue à partir d'une courbe de calibration obtenue par la lecture de solution standard de concentrations connues (généralement $1 \mathrm{ppm}, 5 \mathrm{ppm}$ et $10 \mathrm{ppm}$ ).

Les résultats obtenus, montrent une réduction significative $(p>0,05)$ de la quantité de plomb accumulée dans les feuilles de la laitue comparée à celle de la citronnelle pour les traitements T1 et T2.

Conclusions et application : Les teneurs en cadmium et en plomb dans les feuilles de la laitue ont dépassé les valeurs réglementaires pour l'alimentation. La teneur en plomb dans les feuilles de laitue en culture pure est 9 fois supérieure à la valeur normale tolérée pour les plantes. La co-culture a entrainé une baisse plus ou moins importante de cadmium et de plomb dans les feuilles de la laitue. La promotion des associations laitue-citronnelle peut être envisagée pour la réduction de l'accumulation de plomb par la laitue.

Mots clés : Citronnelle, Laitue, métaux lourds, co-culture 
Evaluation of the transfer level of heavy metals (cadmium, copper, lead and zinc) in Lactuca sativa L. co-cultivated with Cymbopogon citratus (DC) Stapf

\section{ABSTRACT}

Objectives: To evaluate the effects of lettuce and citronella (Cymbopogon citratus (DC) Stapf) associations on the transfer of heavy metals in lettuce (Lactuca sativa L.) organs

Methodology and results: A field trial was conducted. The experimental setup was in completely randomized factorial blocks comprising three treatments and three replicates. Treatments compared pure lettuce (T0) plants with alternating lettuce-lemongrass combinations on the same line (T1) and citronella lettuce where lemongrass plants are placed in interline lettuce (T2). Analyzes were carried out on a flame atomic absorption spectrometer (AAS). The device used is a Perkin Helmer AAnalyst100. The device was managed by a computer. For each element, the concentration value displayed by the computer is obtained from a calibration curve obtained by reading standard solutions of known concentrations (generally 1 ppm, $5 \mathrm{ppm}$ and $10 \mathrm{ppm})$. The results obtained show a significant reduction $(p>0.05)$ of the amount of lead accumulated in the leaves of lettuce compared to that of lemongrass for treatments T1 and T2.

Conclusions and Application: Cadmium and lead levels in lettuce leaves exceeded regulatory values for feed. The lead content in lettuce leaves in pure culture is 9 times higher than the normal tolerated value for plants. The co-cultivation has led to a more or less significant decline of cadmium and lead in the leaves of lettuce. The promotion of intercropping lettuce-citronella may be considered for the reduction of lead accumulation in lettuce plants.

Keywords: Lemongrass, Lettuce, heavy metals, co-cultivation

\section{INTRODUCTION}

Dans bon nombre de pays d'Afrique subsaharienne, le maraichage est une activité qui se pratique beaucoup en zone urbaine et périurbaine. II constitue ainsi une source d'approvisionnement des villes en denrées alimentaires et une source de revenus pour les maraîchers issus principalement de la frange défavorisée de la population (FAO, 2012). Ceci est d'autant plus important que la population urbaine ne cesse de croitre dans ces pays, avec une population vivant en ville qui atteindra 600 millions de personnes à l'horizon 2030 (FAO, 2012). Toutefois, certaines pratiques constatées dans cette activité peuvent susciter des inquiétudes. En effet, divers types de déchets urbains (ménagers, biomédicaux, boues d'épuration) sont principalement utilisés comme fertilisants des sols maraichers en zone urbaine et périurbaine. Si de telles formes de fertilisations des sols permettent le développement des plantes grâce à l'apport de matières organiques, elles sont aussi des sources potentielles voire avérées de contamination de ces mêmes sols (Migeon, 2009), (Kiba et al., 2009) et (llboudo, 2014). Ainsi, diverses substances au nombre desquels les métaux lourds comme le cadmium $(\mathrm{Cd})$, le plomb $(\mathrm{Pb})$, le cuivre $(\mathrm{Cu})$ et le zinc $(\mathrm{Zn})$ voient leurs teneurs augmenter dans les sols (Braud, 2017). Selon l'IFEN, 2002 ; l'épandage de déchets urbains contribue à augmenter de $7 \%$ le $\mathrm{Cd}$ (soit 68 tonnes/an), $5 \%$ le Cu (soit 5.300 tonnes/an), 28\% le Zn (soit 3.200 tonnes/an) et $3 \%$ le $\mathrm{Pb}$ (soit 8.300 tonnes/an) dans les sols en France. L'une des principales voies pour l'imprégnation de ces métaux lourds par l'Homme est la consommation des végétaux cultivés sur ces sols contaminés. Ces végétaux ont la capacité de puiser à travers leurs racines ces substances et les accumuler dans les différents organes (racines, tiges, feuilles) (Mench et Baize, 2004). L'inquiétude découle du fait que ces métaux lourds sont connus pour avoir à certaines concentrations des effets néfastes sur la santé des consommateurs (Nordberg et al., 2004) et (Oskarson et al., 2004) . Parmi ces nombreuses espèces maraichères accumulant les métaux lourds figure la laitue (Zorrig, 2011). Elle a été considérée comme une espèce à risque s'agissant de l'accumulation du cadmium (Zorrig, 2011) et fait partie des principales espèces rencontrées sur les périmètres maraichers du Burkina Faso (Bellwood- 
Howard, 2011). Au Burkina Faso, l'aptitude de certaines espèces locales à accumuler les métaux lourds a été démontrée (Senou, 2014). Au nombre de ces espèces figure Cymbopogon citratus (citronnelle). Cette espèce peut accumuler en moyenne $0,57 \mathrm{mg}$ de Cadmium ; 4,85 mg de cuivre ; $0,012 \mathrm{mg}$ de plomb et $7,53 \mathrm{mg}$ de zinc par $\mathrm{kg}$ de matière sèche (Senou et al., 2014). Par ailleurs, d'autres études ont montré qu'une association culturale entre un hyperaccumulateur des métaux lourds et une espèce sensible peut contribuer à réduire l'accumulation des métaux

\section{MATERIELS ET METHODE}

Présentation de la zone d'étude: L'étude a été réalisée sur le site maraicher de Dogona situé dans la commune urbaine de Bobo-Dioulasso $\left(04^{\circ} 20^{\prime} \mathrm{W}, 11^{\circ}\right.$ $06 ' \mathrm{~N}, 405 \mathrm{~m}$ altitude). Les sols de Bobo-Dioulasso sont du type ferralitique. De texture argileuse kaolinitique dans l'horizon B, ils présentent une infiltration satisfaisante. Les sols dominants sont ceux de type ferrugineux tropicaux sur matériaux divers (sableux, sablo-argileux, argilo-sableux, etc.). Les pH de ces sols varient en général entre 5 et 6,5 (Pallo et al., 2008). Le climat est de type sud-soudanien et la ville de BoboDioulasso est située entre les isohyètes 900 et 1100 $\mathrm{mm}$ caractéristique du climat sud-soudanien (Fontès et Guinko, 1995). On y distingue une saison sèche qui va de novembre à mai et une saison pluvieuse de mai à octobre. Les températures moyennes minimales mensuelles varient de $18^{\circ} \mathrm{C}$ à $25^{\circ}$ en mai. Les températures moyennes maximales mensuelles varient $29^{\circ}$ en aout à $37^{\circ} \mathrm{C}$ en mars. Les vents soufflent à une vitesse moyenne de $2 \mathrm{~m} / \mathrm{s}$ en novembre à $3,5 \mathrm{~m}$ en mai. La moyenne de l'insolation varie de 5,6 heures en aout à 8,7 heures en novembre. La moyenne minimale de l'humidité relative varie de $12 \%$ en février à $66 \%$ en aout.

Matériel végétal : Lactuca sativa L. et Cymbopogon citratus (DC.) Stapf sont des herbacées appartenant respectivement aux familles des Astéracées et des Poacées.

Dispositif expérimental: Le dispositif expérimental est un bloc complet randomisé à trois répétitions comprenant les traitements suivants :

T0 : Culture pure de la laitue avec un écartement de 25 $\mathrm{cm}$ sur les lignes et $25 \mathrm{~cm}$ entre les lignes

T1 : Association laitue + Cymbopogon citratus : plants de laitue alternés avec des plants de citronnelle à des écartements de $10 \mathrm{~cm}$ sur la même ligne. lourds dans la plante sensible. Ainsi, des travaux de (Xiaomei et al., 2005) ont montré que l'hyperaccumulateur du zinc Sedum alfredii permet de réduire de façon significative l'accumulation de ce métal dans les grains de maïs en co-culture. Les recherches ont été menées pour évaluer le niveau de transfert de métaux lourds dans la laitue co-cultivée avec Cymbopogon citratus. L'objectif de cette étude est de réduire le niveau d'accumulation de métaux lourds dans la laitue par C. citratus en co-culture.

T2: Association laitue + Cymbopogon citratus : plants de citronnelle placés dans les interlignes de la laitue avec une distance de $10 \mathrm{~cm}$ avec les lignes de laitue.

Prélèvement des échantillons de sols et d'organes végétaux

Sol : Les échantillons de sol ont été d'abord prélevés juste avant le repiquage des plants afin d'évaluer le niveau de contamination actuelle du sol. A la récolte, des échantillons de sols ont encore été prélevés dans la zone rhizosphérique des plantes dans les différents traitements. Afin de tenir compte de l'hétérogénéité du milieu, un échantillon composite a été constitue à partir d'un mélange de 5 prises élémentaires équipondérales.

Organes végétaux : Les différents échantillons ont été prélevés 40 jours après repiquage des plants de laitue. Pour ce faire, quatre (4) plantes entières ont été récoltées par planche pour constituer un échantillon composite. Les différentes parties végétales (racines, tiges et feuilles) ont été séparées, lavées à l'eau distillée pour éliminer toute contamination externe puis transvasées dans des sacs en plastique bien étiquetés et transportées au laboratoire. Ces échantillons ont été ensuite séchés à température ambiante puis à l'étuve à $70^{\circ} \mathrm{C}$ pendant 24 heures.

Analyse des sols et des organes végétaux

Analyse de métaux lourds dans les sols et dans les organes végétaux : Les teneurs en métaux lourds ont été déterminées dans les sols et dans les organes végétaux de la laitue et de la citronnelle. Les analyses de métaux lourds ont été réalisées au laboratoire de Géochimie de la direction régionale du Bureau des Mines et de la Géologie du Burkina (BUMIGEB). Les différents échantillons ont été d'abord minéralisés à chaud avec $\mathrm{HNO}_{3}$. Pour le dosage des métaux lourds dans les solutions, la lecture a été faite au spectromètre à absorption atomique (SAA) à flamme. L'appareil 
utilisé est un Perkin Helmer AAnalyst100. Le dispositif était géré par un ordinateur. Pour chaque élément, la valeur de la concentration affichée par l'ordinateur est obtenue à partir d'une courbe de calibration obtenue par la lecture de solution standard de concentrations connues (généralement 1 ppm, 5 ppm et 10 ppm).

Analyses chimiques des sols: Les analyses chimiques des échantillons de sol ont été réalisées au laboratoire Eau-Sol-Plantes du département Gestion des Ressources Naturelles et Système de Production (GRN/SP) de l'Institut de l'Environnement et des Recherches agricoles (IN.E.R.A).

La méthodologie de l'extraction des éléments étudiés se présente comme suit :

- $\quad$ Le pHeau a été déterminé par la méthode électronique au pHmètre à électrodes en verre dans un rapport sol/solution de $1: 2,5$.

- $\quad$ Le $P$ assimilable a été déterminé selon la méthode (Bray et Kurtz, 1945). Son extraction est faite avec une solution mixte de chlorure d'ammonium $\left(\mathrm{NH}_{4} \mathrm{Cl}\right)$ et d'acide chlorhydrique $(\mathrm{HCl})$. Les mesures sont effectuées au spectrophotomètre à $880 \mathrm{~nm}$ de longueur d'onde en utilisant le molybdate d'ammonium $\left(\mathrm{NH}_{4} 6 \mathrm{Mo}_{7} .4 \mathrm{H}_{2} \mathrm{O}\right)$.

- Le carbone du sol a été déterminé par la méthode de (Walkley et Black, 1934) (oxydation à froid du carbone du sol au bichromate de potassium

\section{RESULTATS}

Paramètres chimiques des sols avant et après culture: Les résultats analytiques des paramètres chimiques des sols sont consignés dans le Tableau 1. Les valeurs de $\mathrm{pH}$ sont comprises entre 6,73 et 7,35 respectivement pour les traitements T2 et TO. La valeur de $\mathrm{pH}$ n'a pas significativement modifiée quel que soit le model d'association. Les teneurs en carbone, phosphore assimilable, azote et matière organique du sol ont baissé dans les traitements en co-culture (T1 et T2) tandis que le P-total et le K-total ont subi une
$\left(\mathrm{K}_{2} \mathrm{Cr}_{2} \mathrm{O}_{7}\right) 1 \mathrm{~N}$ dans un milieu acide sulfurique concentré).

- $\quad$ La capacité d'échange cationique (CEC) a été déterminée par la méthode de Metson à l'acétate d'ammonium. Un échantillon de $25 \mathrm{~g}$ de sol est percolé avec $75 \mathrm{ml}$ d'une solution molaire d'acétate d'ammonium.

- $\quad$ Pour la détermination du P-total, de N-total et du K-total, les différents échantillons ont préalablement été minéralisés à chaud avec un mélange $\mathrm{H}_{2} \mathrm{SO}_{4}$-Se$\mathrm{H}_{2} \mathrm{O}_{2}$. Les teneurs en $\mathrm{N}$-total et $\mathrm{P}$-total ont été par la suite déterminées dans les minéralisâtes à l'aide d'un colorimètre automatique. Quant à la teneur en K-total, elle a été déterminée au moyen d'un photomètre de flamme.

Traitement des données: L'analyse statistique des données a été réalisée avec le logiciel $R$ version 3.5.1. (R Core Team (2018)). Un test de normalité a été effectué et suivant le résultat les variables ont été soumises soit à un test non paramétrique (test de Kruskal-Wallis), soit à une ANOVA. La comparaison des moyennes pour l'ANOVA a été faite par le test de Tukey. Le seuil de signification pour l'ensemble des tests était de 5\%.

augmentation. Cependant, cette baisse n'a été significative que pour la matière organique et la phosphore assimilable. Le rapport $\mathrm{C} / \mathrm{N}$ a par contre baissé de 1 unité avec la co-culture. Quel que soit le traitement, la CEC a baissé avec la présence de culture. Elle est plus faible dans les sols en co-culture que dans les sols en culture pure de la laitue. La CEC des sols avant culture est 3 fois plus élevée que celle des sols avec culture. 
Tableau 1 : Paramètres chimiques du sol avant et après culture

\begin{tabular}{cccccccccc}
\hline Traitements & \multicolumn{7}{c}{ Paramètres chimiques du sol } \\
\hline pHeau & $\begin{array}{c}\text { Carbone } \\
(\%)\end{array}$ & $\begin{array}{c}\text { CEC } \\
(\mathrm{méq} / 100 \mathrm{~g})\end{array}$ & $\mathrm{M} . \mathrm{O}(\%)$ & P-Total $(\mathrm{mg} / \mathrm{kg})$ & $\begin{array}{c}\text { P-Assimilable } \\
(\mathrm{mg} / \mathrm{kg})\end{array}$ & $\mathrm{K}$-total $(\mathrm{mg} / \mathrm{kg})$ & $\mathrm{N}(\%)$ & $\mathrm{C} / \mathrm{N}$ \\
\hline TO/AO & $7,01 \mathrm{a}$ & $1,91 \mathrm{a}$ & $16,80 \mathrm{a}$ & $3,32 \mathrm{a}$ & $595,5 \mathrm{a}$ & $62,14 \mathrm{~b}$ & $1790,2 \mathrm{a}$ & $0,17 \mathrm{a}$ & $12 \mathrm{a}$ \\
\hline T0 & $7,35 \pm 0,05 \mathrm{a}$ & $1,64 \pm 0,24 \mathrm{a}$ & $5,33 \pm \mathrm{b}$ & $2,76 \pm 0,23 \mathrm{ab}$ & $557,93 \pm 35,79 \mathrm{a}$ & $29,05 \pm 1,86 \mathrm{a}$ & $2304,03 \pm 60,82 \mathrm{~b}$ & $0,18 \pm 0,12 \mathrm{a}$ & $12,67 \pm 0,58 \mathrm{a}$ \\
\hline T1 & $7,18 \pm 0,76 \mathrm{a}$ & $1,77 \pm 0,24 \mathrm{a}$ & $4,81 \pm 0,85 \mathrm{~b}$ & $2,66 \pm 0,34 \mathrm{~b}$ & $608,3 \pm 17,2 \mathrm{a}$ & $56,71 \pm 11,61 \mathrm{~b}$ & $2100,1 \pm 88,1 \mathrm{~b}$ & $0,16 \mathrm{a}$ & $11 \pm 1 \mathrm{a}$ \\
\hline T2 & $6,73 \pm 0,35 \mathrm{a}$ & $1,36 \pm 0,27 \mathrm{a}$ & $4,61 \pm 0,20 \mathrm{~b}$ & $2,53 \pm 0,40 \mathrm{~b}$ & $620,83 \pm 61,32 \mathrm{a}$ & $53,36 \pm 6,08 \mathrm{~b}$ & $2249,97 \pm 181,14 \mathrm{~b}$ & $0,16 \pm 0,01 \mathrm{a}$ & $11,33 \pm 0,58 \mathrm{a}$ \\
\hline Probabilité & 0,37 & 0.07 & $0,017^{*}$ & $0,01^{*}$ & 0,25 & $0,001^{* *}$ & $0,001^{* *}$ & 0,95 & 0,05
\end{tabular}

TO/A0 : sol avant installation des cultures, T0 : sol culture pure de laitue, T1 : sol culture de la citronnelle alternée à la laitue sur même ligne, T2 : sol culture de la citronnelle entre les lignes de laitue. Les lettres identiques dans une colonne entre deux valeurs indiquent qu'elles ne sont pas significativement différentes au seuil de $5 \%$, 
Teneurs en métaux lourds des sols avant et après culture: Les teneurs initiales en métaux lourds et celles après culture sont consignées dans le Tableau 2. Les sols de départ présentent des teneurs en zinc (130, $63 \mathrm{mg} / \mathrm{kg}$ ) plus élevés que les autres métaux lourds $(0,24 \mathrm{mg} / \mathrm{kg})$. Le cadmium est le métal qui a la plus faible teneur. La même tendance est observée dans les sols rhizosphériques des plantes après culture quel que soit le traitement. De façon générale, les teneurs sont plus élevées dans les sols après cultures comparés aux sols après culture. Des différences significatives sont notées entre les teneurs en métaux lourds des sols avant culture et des sols après culture. Ces différences sont observées pour le Cu avec le traitement T1, pour le $\mathrm{Pb}$ avec le traitement $\mathrm{T} 1$ et $\mathrm{T} 2$ et avec le $\mathrm{Zn}$ pour tous les trois traitements (T1, T2, T3). La teneur en $\mathrm{Cd}$ dans les sols avant et après culture n'a pas montré de différence significative quel que soit le traitement.

Tableau 2 : Teneurs en métaux lourds dans les sols avant et après culture

\begin{tabular}{lllll}
\hline Traitements & \multicolumn{4}{l}{ Teneurs en métaux lourds $(\mathbf{m g} / \mathbf{k g}$ de matière sèche) $\mathbf{n}=\mathbf{3}$} \\
\hline & $\mathrm{Cd}$ & $\mathrm{Cu}$ & $\mathrm{Pb}$ & $\mathrm{Zn}$ \\
\hline T0/A0 & $0,24 \mathrm{a}$ & $11,32 \mathrm{a}$ & $27.84 \mathrm{a}$ & $130,63 \mathrm{a}$ \\
\hline T0 & $0,21 \pm 0.17 \mathrm{a}$ & $13,21 \pm 3,91 \mathrm{a}$ & $35,07 \pm 13,44 \mathrm{a}$ & $153,43 \pm 15,88 \mathrm{~b}$ \\
\hline Probabilité & 0,8 & 0,44 & 0,49 & $0,03^{*}$ \\
\hline T0/A0 & & & & \\
\hline T1 & $0,24 \mathrm{a}$ & $11,32 \mathrm{a}$ & $27.84 \mathrm{a}$ & $130,63 \mathrm{a}$ \\
\hline Probabilité & $0,28 \pm 0,19 \mathrm{a}$ & $15,94 \pm 2,93 \mathrm{~b}$ & $32,27 \pm 2,37 \mathrm{a}$ & $364,33 \pm 298,36 \mathrm{~b}$ \\
\hline T0/A0 & 0,49 & $0,04^{*}$ & $0.03^{*}$ & $0,04^{*}$ \\
\hline T2 & & & & \\
\hline Probabilité & $0,24 \mathrm{a}$ & $11,32 \mathrm{a}$ & $27.84 \mathrm{a}$ & $130,63 \mathrm{a}$ \\
\hline
\end{tabular}

TO/A0 : sol avant installation des cultures, T0 : sol culture pure de laitue, T1 : sol culture de la citronnelle alternée à la laitue sur même ligne, T2: sol culture de la citronnelle entre les lignes de laitue. Les lettres identiques dans une colonne entre deux valeurs indiquent qu'elles ne sont pas significativement différentes au seuil de $5 \%, \quad n:$ nombre de répétitions *valeur de probabilité significative.

Teneurs en métaux lourds dans les organes de la laitue en culture pure: Les niveaux des métaux lourds étudiés dans les différents organes (racines, tiges et feuilles) de laitue sont présentés dans le Tableau 3. Quel que soit l'organe végétal, le zinc est le métal plus accumulé suivi du plomb. La racine est l'organe végétal qui a le plus accumulé le Zinc, le plomb et le cuivre avec des teneurs respectivement plus élevées en zinc $(108,74 \mathrm{mg} / \mathrm{kg})$, plomb $(18,09$ $\mathrm{mg} / \mathrm{kg}$ ) et cuivre $(13,19 \mathrm{mg} / \mathrm{kg})$. Les teneurs en Cu et
$\mathrm{Pb}$ ont varié de manière très significative $(\mathrm{P}>0,001)$ entre les différents organes. La plus forte teneur en cadmium $(0,94 \mathrm{mg} / \mathrm{kg})$ a été notée dans les feuilles. Cependant, aucune différence significative n'a été observée entre les organes. Par contre, des différences significatives ont été observées pour les teneurs en zinc, plomb et cuivre dans les différents organes de la laitue.

Tableau 3 : Concentrations des différents métaux lourds dans les organes de la laitue

\begin{tabular}{lllll}
\hline Organes & \multicolumn{4}{c}{ Teneurs en métaux lourds $\mathbf{( m g / k g ~ M S )}$} \\
\hline Racines & $\mathrm{Cd}$ & $\mathrm{Cu}$ & $\mathrm{Pb}$ & $\mathrm{Zn}$ \\
\hline Tiges & $0,70 \pm 0,20 \mathrm{a}$ & $13,19 \pm 4,93 \mathrm{a}$ & $18,09 \pm 2,89 \mathrm{a}$ & $108,74 \pm 8,65 \mathrm{a}$ \\
\hline Feuilles & $0,35 \pm 0,13 \mathrm{a}$ & $0,38 \pm 0,66 \mathrm{~b}$ & $4,11 \pm 2,14 \mathrm{~b}$ & $65,08 \pm 38,24 \mathrm{~b}$ \\
\hline Probabilité & $0,94 \pm 1,16 \mathrm{a}$ & $2,37 \pm 0,39 \mathrm{~b}$ & $2,7 \pm 1,6 \mathrm{~b}$ & $62,20 \pm 7,66 \mathrm{~b}$ \\
\hline
\end{tabular}


T0 : culture pure de laitue, T1 : citronnelle alternée avec la laitue sur la même ligne, T2 : co-culture de citronnelle entre les lignes de laitue. Les lettres identiques dans une colonne entre deux valeurs indiquent qu'elles ne sont pas significativement différentes au seuil de $5 \%$. *valeur significative au seuil de probabilité de $5 \%$

\section{Comparaison des teneurs en métaux lourds dans les organes de la laitue et de la citronnelle en co- culture}

Les comparaisons entre la laitue et la citronnelle pour l'accumulation de métaux lourds sont présentées à travers les Figures 1, 2, 3 et 4. Ces comparaisons ont été faites pour les quatre métaux lourds et avec deux organes végétaux (racine et feuille).

- Plomb: La figure 1 montre des teneurs moyennes en $\mathrm{Pb}$ significativement plus élevées $(P<0,05)$ dans les feuilles de citronnelle par rapport à celles de la laitue et ce, quel que soit le type d'association du type d'association. Quant aux teneurs en $\mathrm{Pb}$ dans les racines, les valeurs ne montrent aucune différence significative (Figure 1).

- Zinc: La concentration en zinc dans les racines de la laitue a été significativement supérieure à celle de la citronnelle lorsque les deux plantes ont été cultivées de façon alternée sur la même ligne (T1) (Figure 2). Pour le traitement T2 (plants de citronnelle placés dans les interlignes de la laitue), l'accumulation est plus élevée dans la laitue quel que soit l'organe végétal. Toutefois, les teneurs en zinc n'ont pas varié significativement dans les feuilles entre les deux espèces et cela pour les deux traitements (T1 et T2) (Figure 2).

- Cuivre : La teneur en cuivre $(\mathrm{Cu})$ a été plus élevée dans la laitue comparée à la citronnelle quel que soit le traitement et l'organe végétal. Cependant, la différence significative a été notée dans feuilles pour le traitement T2 et dans les racines pour le traitement $\mathrm{T} 1$ (Figure 3).

- Cadmium: Les résultats présentés dans la figure 4 indiquent que la laitue a accumulé plus de cadmium (Cd) que la citronnelle quel que soit le traitement et l'organe végétal. Dans les racines, la différence est significative pour les deux traitements (Figure 4b). Par contre, dans les feuilles elle est significative que pour le traitement T2 (Figure 4a).

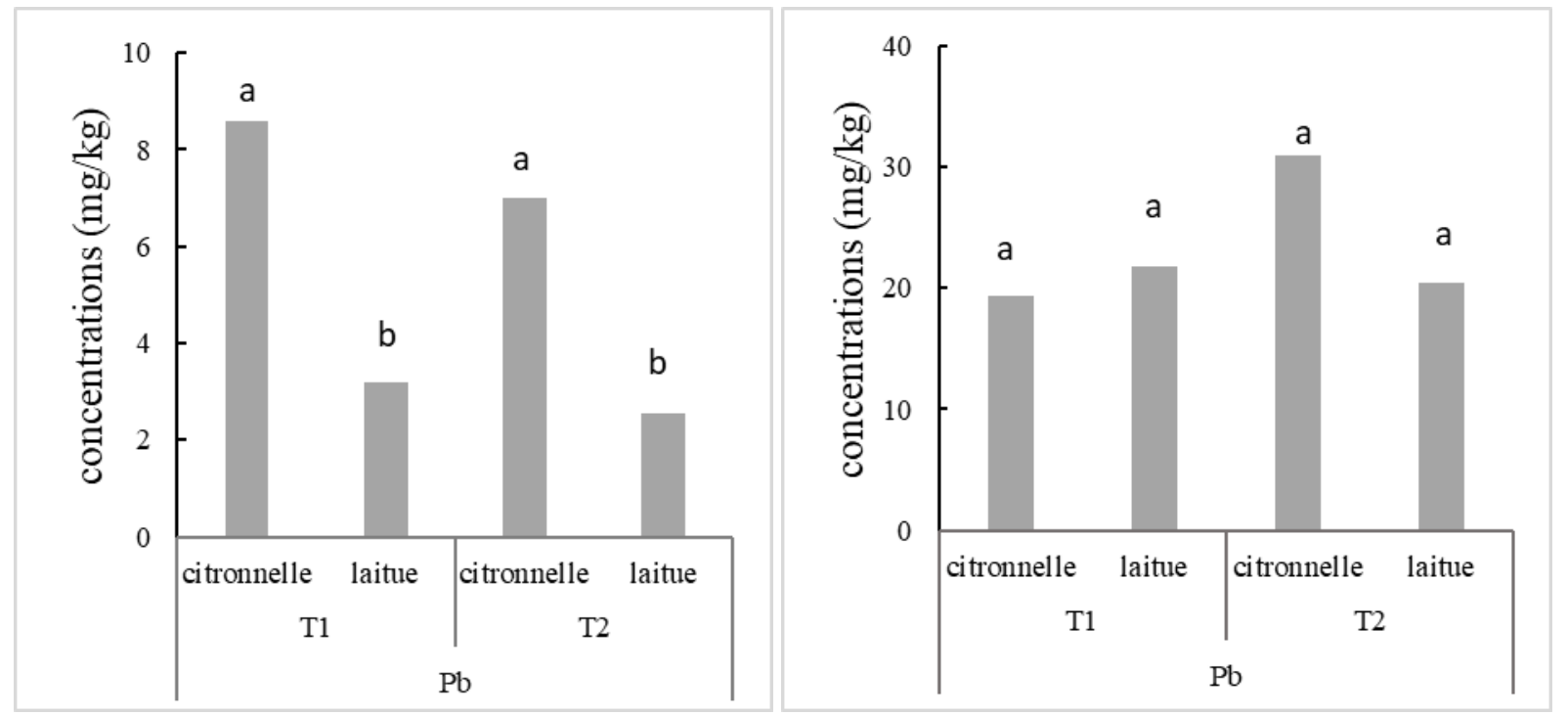

Figure 1 a : Teneurs foliaires en Plomb

Figure $1 \mathrm{~b}$ : Teneurs racinaires en Plomb

Figure 1 : Comparaison de la teneur en plomb $(\mathrm{Pb})$ accumulée par la laitue et la citronnelle. 

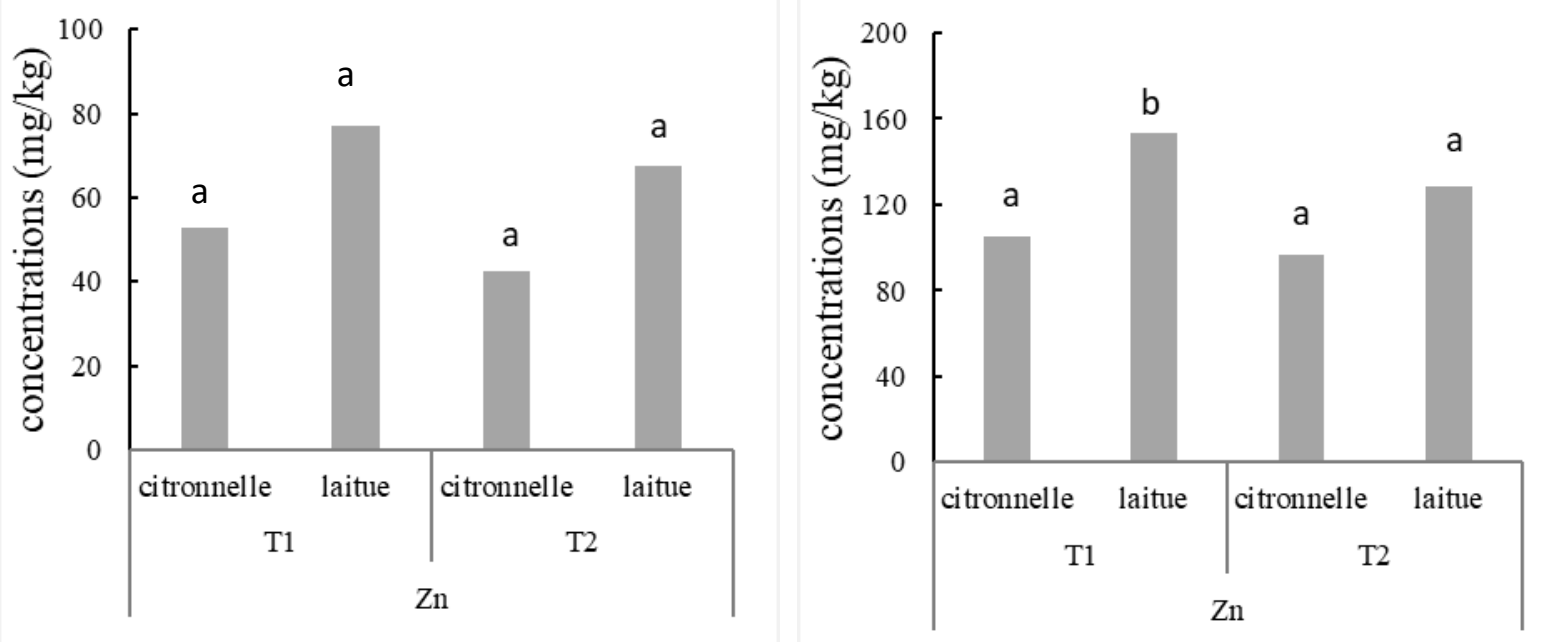

Figure $2 \mathbf{a}$ : Teneurs foliaires en zinc. Figure $\mathbf{2 b}$ : Teneurs racinaires en zinc

Figure 2 : Comparaison de la teneur en zinc (Zn) accumulée par la laitue et la citronnelle.

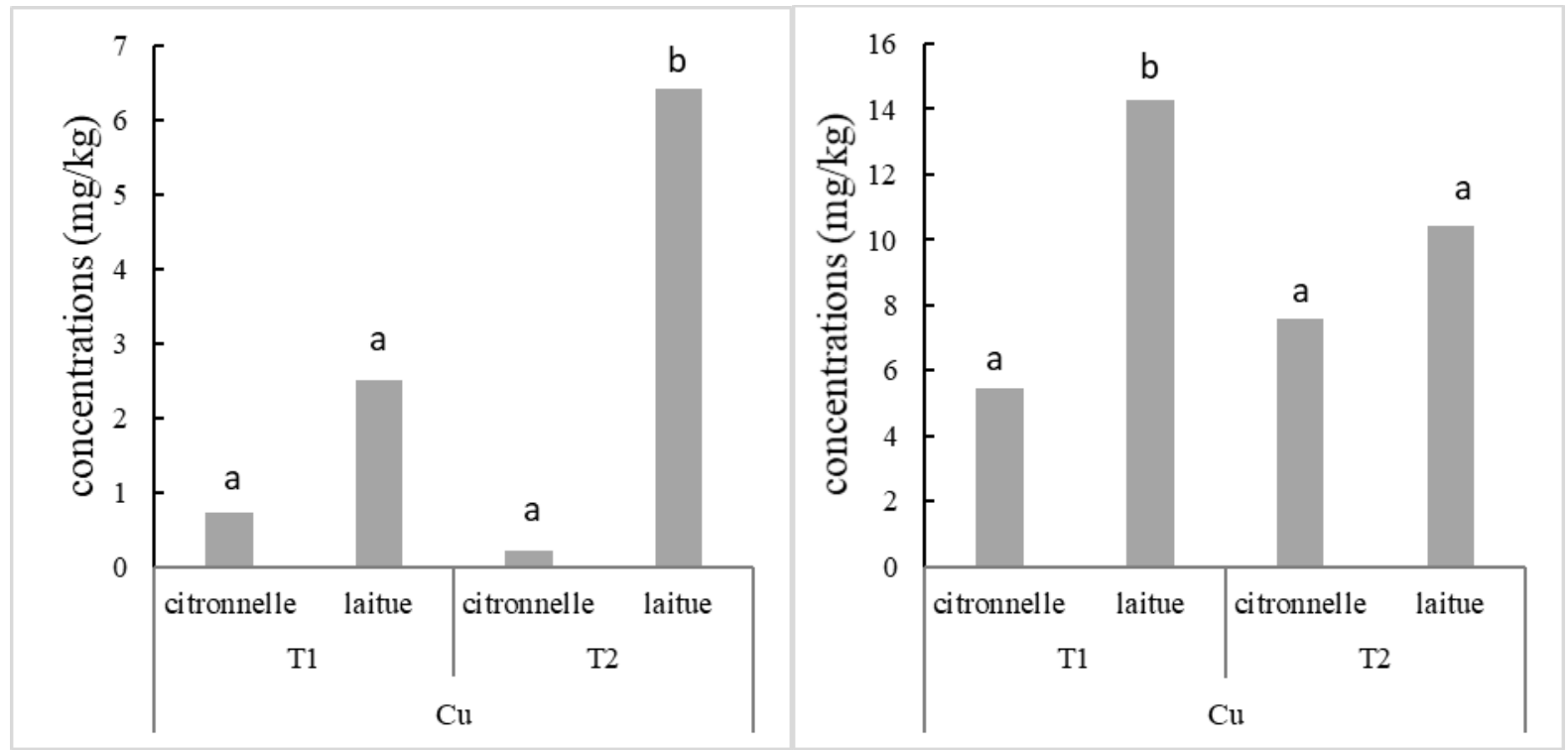

Figure 3a : Teneurs foliaires en cuivre.

Figure 3b : Teneurs racinaires en cuivre

Figure 3 : Comparaison de la teneur en cuivre $(\mathrm{Cu})$ accumulée par la laitue et la citronnelle. 


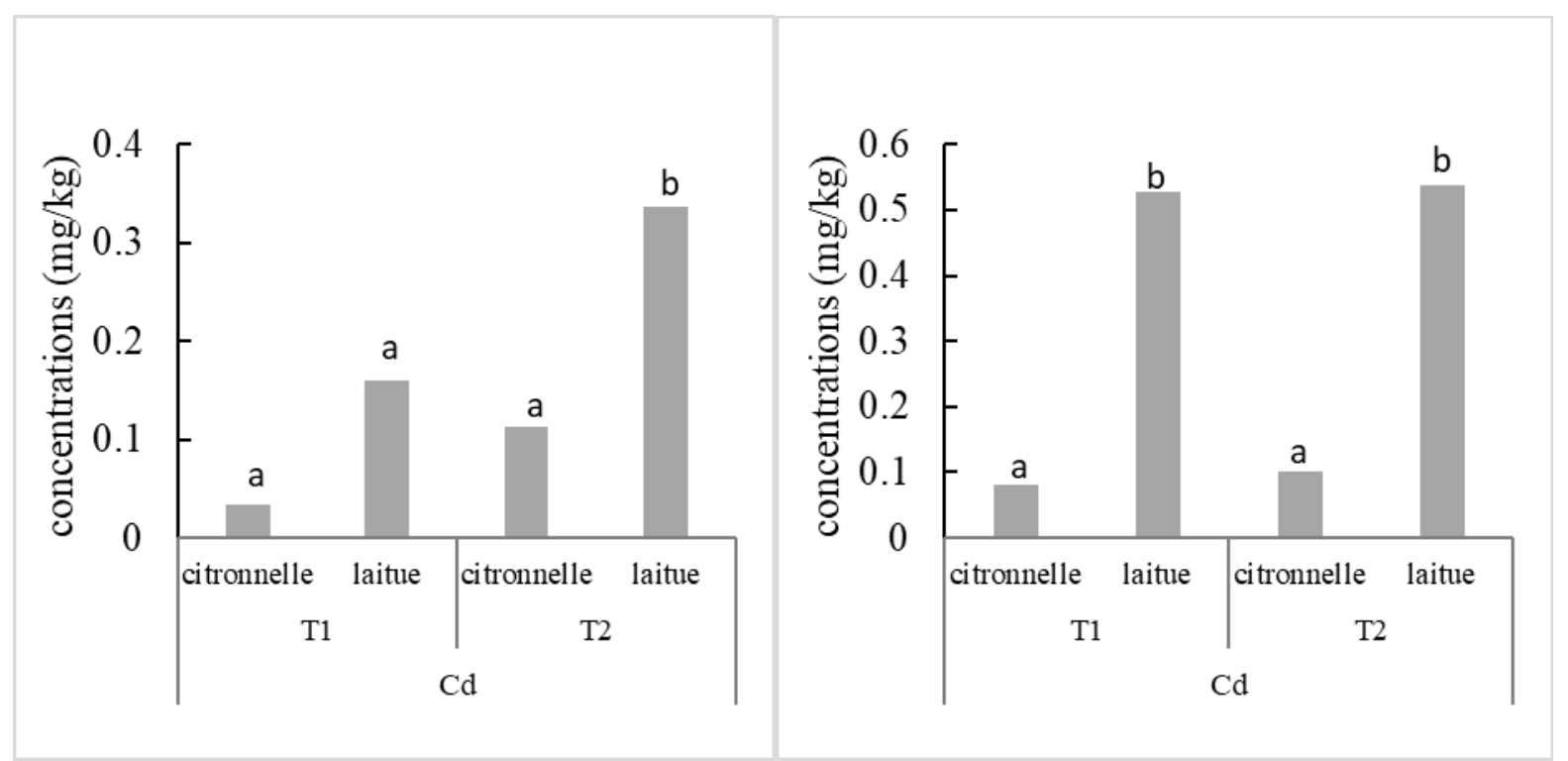

Figure $4 \mathrm{a}$ : Teneurs foliaires en cadmium. Figure $4 \mathrm{~b}:$ Teneurs racinaires en cadmium Figure 4 : Comparaison de la teneur en cadmium accumulée par la laitue et la citronnelle.

\section{DISCUSSION}

Paramètres chimiques du sol avant et après culture: A l'issu de ces travaux, il y a eu une baisse significative de la matière organique dans les sols rhizosphériques. Cela pourrait s'expliquer par la minéralisation de cette fraction du sol. Parmi les paramètres chimiques déterminés figurait le $\mathrm{pH}$ du sol qui est demeuré proche de la neutralité pendant la culture. La faible minéralisation de la matière organique pendant la culture de la citronnelle et de laitue aurait une faible influence sur la variation du $\mathrm{pH}$. La diminution de la capacité d'échange cationique d'une part et l'augmentation des teneurs en potassium-total d'autre part, seraient liées à la minéralisation de la matière organique dans les sols rhizosphériques après culture. Selon (Bewket et Stroosnijder, 2003), la capacité d'échange cationique est généralement déterminée par les teneurs en matières organiques et minéraux argileux dans les sols. Les travaux (Ondo, 2011) ont également montré une corrélation forte et positive $(0,712)$ entre la teneur en matière organique $d u$ sol et la capacité d'échange cationique des sols au niveau des périmètres maraichers. La matière organique améliore en plus les propriétés biologiques et physico-chimiques des sols, et constitue une source d'éléments nutritifs (Potassium-Total) (Mirsa, 2005). Les teneurs en azote total et en phosphore total n'ont cependant pas évolué significativement pendant la culture. La stabilité des teneurs en azote et en phosphore pourrait être liée au prélèvement de ces éléments par les plantes. La baisse significative de la teneur en phosphore assimilable au niveau de T0, serait due au pH du sol, car le $\mathrm{pH}$ influence considérablement la disponibilité du phosphore. Le rapport $\mathrm{C} / \mathrm{N}$ traduit le niveau de minéralisation des matières organiques dans les sols. Les valeurs de ce rapport obtenu au niveau des sols correspondent à celles indiquant une bonne minéralisation de la matière organique, c'est-à-dire comprises entre 8 et 12 (Genot, 2007).

Teneurs en métaux lourds des sols avant et après culture: Les résultats indiquent que les teneurs du $\mathrm{Pb}, \mathrm{du} \mathrm{Cu}$ et du $\mathrm{Cd}$ avant culture ont sont en légèrement inférieures aux valeurs moyennes mondiales des sols non contaminés ( $\mathrm{Cd} 0,35 \mathrm{mg} / \mathrm{kg}$; Cu $30 \mathrm{mg} / \mathrm{kg}$; Pb $35 \mathrm{mg} / \mathrm{kg}$; Zn $90 \mathrm{mg} / \mathrm{kg}$; Bowen, 1979). En effet, les seuils tolérables des métaux lourds dans le sol contaminés sont de $0,35 \mathrm{mg} / \mathrm{kg}$ pour le cadmium ; $30 \mathrm{mg} / \mathrm{kg}$ pour le cuivre ; $35 \mathrm{mg} / \mathrm{kg}$ pour le plomb et $90 \mathrm{mg} / \mathrm{kg}$ pour le zinc (Bowen, 1979). Quant au zinc, sa teneur a excédé le seuil tolérable de 90 $\mathrm{mg} / \mathrm{kg}$ (Bowen, 1979). Par ailleurs, les teneurs en plomb, cuivre zinc ont augmenté dans les sols après culture. Cette hausse pourrait s'expliquer par un enrichissement des sols en métaux lourds pendant les différentes opérations culturales. Les engrais utilisés pour la fertilisation, les produits phytosanitaires, l'eau d'arrosage pourraient contribuer à enrichir les sols en métaux lourds.

Teneurs en métaux lourds dans les organes de la laitue en culture pure: Nos résultats ont montré que 
la laitue en culture pure a accumulé les métaux lourds dans ses différents organes. Toutefois, les teneurs ont été inférieures aux valeurs limites au-dessus desquelles des phytotoxicités sont possibles (5-30 $\mathrm{mg} / \mathrm{kg}$ pour $\mathrm{Cd}, 20-200 \mathrm{mg} / \mathrm{kg}$ pour $\mathrm{Cu}, 30-300 \mathrm{mg} / \mathrm{kg}$ pour $\mathrm{Pb}, 100-400 \mathrm{mg} / \mathrm{kg}$ pour $\mathrm{Zn}$ ) selon (Larcher, 2003). La valeur moyenne $(>2 \%)$ de la teneur en matière organique des sols et les $\mathrm{pH}$ proches de la neutralité auraient favorisé les faibles niveaux d'accumulation (Lair et al., 2007). Dans le sol, les pH acides favorisent le transfert des métaux lourds vers la plante tandis que les $\mathrm{pH}$ proches de la neutralité permettent leur rétention par la matière organique (Lair et al., 2007). Cependant, les teneurs foliaires de la laitue en cadmium et plomb ont dépassé les valeurs réglementaires pour l'alimentation qui sont de 0,2 $\mathrm{mg} / \mathrm{kg}$ pour cadmium et $0,3 \mathrm{mg} / \mathrm{kg}$ pour plomb (FAO/OMS, 2015). Par exemple, la teneur en plomb dans les feuilles de laitue en culture pure a été 9 fois supérieure à la valeur normale tolérée. Cela pourrait représenter un risque pour la santé des consommateurs. En effet, les métaux lourds absorbés par les végétaux entrent dans la chaîne alimentaire et entraînent un phénomène de bio-concentration à chaque passage dans le maillon trophique supérieur (Gonzales et al., 2008), (McLean et al., 2009). Cette accumulation de métaux lourds s'avère dangereuse pour la santé Des résultats similaires ont été obtenus avec des teneurs en cadmium et plomb respectivement de $0,31 \mathrm{mg} / \mathrm{kg}$ et $24,36 \mathrm{mg} / \mathrm{kg}$ dans les feuilles de la laitue (Seka et al., 2006). Ces résultats corroborent ceux de (Donkor et al., 2017) qui ont montré des teneurs en métaux lourds excédant les normes dans des cultures de la laitue.

Comparaison des teneurs en métaux lourds dans les organes de la laitue et de la citronnelle en coculture :

De cette étude, il ressort que les associations entre la laitue et la citronnelle n'ont pas permis de réduire le niveau d'accumulation de cuivre et de zinc au niveau des différents organes de la laitue. Par contre, cette étude a observé une baisse plus ou moins importante de la teneur de cadmium et de plomb dans les feuilles de la laitue. Toute chose qui suggère que l'association laitue-citronnelle pourrait effectivement réduire le

\section{CONCLUSION}

Ce travail avait pour objectif principal de réduire le niveau d'accumulation de métaux lourds dans la laitue co-cultivée avec par la citronnelle. II ressort que la laitue en culture pure accumule les métaux lourds dans niveau de transfert du cadmium et de plomb dans les feuilles de laitue. En effet, les métaux lourds comme le cadmium, le nickel, le plomb ou le mercure ne jouent aucun rôle dans la croissance et le développement des plantes (Mendoza-Cózatl et Moreno-Sanchez, 2005). Ils sont dits non essentiels et sont toxiques à l'état de traces (Mendoza-Cózatl et Moreno-Sanchez, 2005).Concernant la citronnelle, les résultats ont montré qu'elle a accumulé les métaux lourds dans chacun de ses organes pour les deux types d'association dans cette étude. Les teneurs en cadmium, cuivre et zinc au niveau des racines et des feuilles de la citronnelle dans les deux types d'associations sont inférieures à celles trouvées par Senou et al. (2018). Les travaux de Senou et al. (2018) ont montré des concentrations beaucoup plus élevées dans les mêmes organes avec la citronnelle cultivée trois mois durant sur le même type de sol auxquels ont été ajoutés des déchets urbains solides. Par contre, pour ce qui est du $\mathrm{Pb}$, les concentrations obtenues dans la présente étude sont supérieures aux valeurs de obtenues par (Senou et al., 2018). Ces différences pourraient s'expliquer d'une part par la variation des teneurs initiales dans les sols et des paramètres du sol susceptibles de modifier la biodisponibilité des métaux lourds dans le sol. Les concentrations en plomb accumulées ont été plus élevées dans la citronnelle par rapport à la laitue au niveau des feuilles. Ce résultat pourrait se traduire par la grande aptitude de citronnelle à la translocation du plomb vers les organes aériens (Senou et al., 2018). La citronnelle est connue comme une espèce efficace pour ce qui est du transfert du plomb des racines vers les parties aériennes (Gautam et al., 2017). Cependant, les résultats montrent qu'en co-culture laitue-citronnelle, la laitue a accumulé le plus de zinc, de cuivre et de cadmium comparativement à la citronnelle. Les travaux de Agbossou et al., 2003) ont montré que la croissance rapide de la laitue accélère le prélèvement des nutriments nécessaires à sa croissance dans le sol, notamment le zinc et cuivre. En outre, les résultats de ADEME (1990) ont montré la grande capacité des espèces à croissance rapide telle que la laitue à accumuler le zinc, le cadmium et le cuivre.

ses différents organes. Les teneurs en cadmium et en plomb dans les feuilles ont dépassé les valeurs réglementaires pour l'alimentation. Ces valeurs sont de $0,2 \mathrm{mg} / \mathrm{kg}$ pour cadmium et $0,3 \mathrm{mg} / \mathrm{kg}$ pour plomb. La 
teneur en plomb dans les feuilles de laitue en culture pure est 9 fois supérieure à la valeur normale tolérée pour les plantes. L'association entre la laitue et la citronnelle n'a pas permis de réduire le niveau d'accumulation de cuivre et de zinc au niveau des différents organes de la laitue. Toutefois, une baisse plus ou moins importante de la teneur de cadmium et

\section{REMERCIEMENTS}

Nous exprimons nos sincères remerciements à la Direction Régionale du Bureau des Mines et de

\section{REFERENCES}

ADEME, 1990. Connaissance et maîtrise des aspects sanitaires de l'épandage des boues. France.

Agbossou, K., Sanny, M. S., Zokpodo, B., Ahamide, B. et Guedegbe, H. J. 2003. Evaluation qualitative de quelques légumes sur le périmètre maraîcher de houéhiyo, Cotonou au sud-Bénin. Bulletin de la Recherche Agronomique du Bénin, (42), pp.1-12

Bellwood-Howard I., Häring V., Karg H., Roessler R., Schlesinger J., Shakya M. 2015. Characteristics of urban and peri-urban agriculture in West Africa: results of an exploratory survey conducted in Tamale (Ghana) and Ouagadougou (Burkina Faso). p. 163

Bewket W., Stroosnijder L. 2003. Effects of agroecological land use succession on soil properties in Chemical watershed, Blue Nile basin, Ethiopia. Geoderma, 111, p.85-98

Bowen H.J.M. 1979. Environmental Chemistry of the Elements. Academic Press, New York, pp. 4962

Braud A. 2007. Procédé de phytoextraction couplé à la bioaugmentation d'un sol agricole polycontaminé par du chrome, du mercure et du plomb. Thèse de doctorat, Université de Haute-Alsace, p. 254

Bray R. H., Kurtz L. T. 1945. Determination of total, organic, and available forms of phosphorus in soils. Soil Science, 59 pp. 39-45

Donkor, A., Fordjour, L. A., Tawiah, R., Asomaning, W., Dubey, B., Osei-fosu, P., Ziwu, C. et Mohammed, M. 2017. Evaluation of trace metals in vegetables sampled from farm and market sites of Accra Metropolis, Ghana. International Journal of Environmental Studies, 74 (2), pp. 315-324

FAO, 2012. Growing greener cities, Rome. p. 116 de plomb dans les feuilles de la laitue est observée. Cela suggère que l'association laitue-citronnelle pourrait être efficace pour réduire le niveau de transfert du cadmium et de plomb dans les feuilles de laitue. On peut conclure que l'association laitue-citronnelle peut être envisagée pour la réduction de l'accumulation de plomb dans les organes de la laitue.

Géologie du Burkina Faso pour son apport dans les analyses de métaux lourds.

FAO/OMS, 2015. Norme générale pour les contaminants et les toxines présents dans les produits de consommation humaine et animale, CODEX STAN. p. 64

Fontès J., Guinko S. 1995. Carte de la végétation et du sol du Burkina Faso. Notice explicative. Ministère de la coopération française. Projet campus, p. 67

Gautam M., Pandey D., Agrawal M. 2017. Phytoremediation of metals using lemongrass (Cymbopogon citratus (DC.) Stapf) grown under different levels of red mud in soil amended with biowastes. International Journal of Phytoremediation, 19 (6), pp. 555-562

Genot V., Colinet G., Bock L. 2007. Fertilité des sols agricoles et forestiers en région wallonne, Rapport FUSAG, p. 75

Gonzales X.I., Aboal J.R., Fernandez J.A., Carballeira A. 2008. Heavy metal transfers between trophic compartments in different ecosystems in Galicia (Northwest Spain): Essential elements. Archives of Environmental Contamination and Toxicology 55, pp. 691-700

IFEN, 2002. Principales causes d'apport de métaux lourds dans les sols d'usage agricole. L'environnement en France. Paris, Dunod. p. 83

Ilboudo L.J.T. 2014. Effet de différents types de déchets urbains sol ides de la ville de BoboDioulasso sur la disponibilité et la distribution verticale de métaux lourds dans le sol. Mémoire. Université Polytechnique de BoboDioulasso, p. 51

Kiba D. I., Lompo, F., Compaore, E., Randriamanantsoa, L., Sedogo, P. M., Frossard, E. 2009. A decade of non-sorted solid urban wastes inputs safely increases sorghum yield in periurban areas of Burkina 
Faso. Acta Agriculturae Scandinavica Section B: Soil and Plant Science, 62 (1), pp.59-69

Lair G. J., Gerzabek M. H., Haberhauer G. 2007. Sorption of heavy metals on organic and inorganic soil constituents. Environmental Chemistry Letters, 5 (1), pp. 23-27

Larcher W. 2003. Physiological plant ecology. 4e éd. Springer. p. 513

McLean C.M., Koller C.E., Rodger J.C., MacFarlane G.R. 2009. Mammalian hair as an accumulative bioindicator of metal bioavailability in Australian terrestrial environments. Science of the Total Environment 407 (11): pp. 3588-3596

Mench M., Baize D. 2004. Contamination des sols et de nos aliments d'origine végétale par les éléments en traces mesures pour réduire l'exposition. Courrier de l'environnement de I'INRA, 52, pp.31-56

Mendoza-Cózatl D.G., Moreno-Sanchez R. 2005. Cd2+ transport and storage in the chloroplast of Euglena gracilis. Biochimica et Biophysica Acta 1706 : pp. 88-97

Migeon A. 2009. Etude de la variabilité naturelle dans la réponse du peuplier aux métaux: bases physiologiques et exploitation en phytoremédiation. Thèse, Université Henri Poincaré, Nancy-Université, p. 345

Mirsa R. V., Roy R. N., Hiraoka H. 2005. Méthodes de compostage au niveau de l'exploitation. Documents de travail sur les terres et les eaux 2. Organisation des nations Unies pour l'Alimentation et l'Agriculture, Italie, Rome, p. 35

Nordberg M., Nordberg G., Jin T. 2004. Health impacts of cadmium exposure and its prevention. BioMetals, 17, pp.483-484

Ondo J.A. 2011.Vulnérabilité des sols maraîchers du Gabon (région de Libreville) : acidification et mobilité des éléments métalliques. Thèse, Université de Provence (France). p. 304

Oskarson A., Widell A., Olsson I., Graw K. P. 2004. Cadmium in food chain and health effects in sensitive population groups. BioMetals, 17, pp.531-534

Pallo F.J.P., Sawadogo N., Sawadogo L., Sedogo P. M. 2008. Statut de la matière organique des sols dans la zone sudsoudanienne au Burkina Faso. Biotechnol Agron Soc Environ, 12: pp. 291-301

Seka Y. J., Yapo O. B., Yapi D. A. C. 2015. Heavy Metals Contamination in Lactuca sativa $L$. (Lettuce) from Two Agricultural Sites of Abidjan. International Journal of Pure and Applied Sciences, 27 (2), pp. 59-64

Senou I. 2014. Phytoextraction du cadmium, du cuivre, du plomb et du zinc par cinq espèces végétales (Vetiveria nigritana (Benth.), Oxytenanthera abyssinica (A. Rich.) Munro, Barleria repens (Ness), Cymbopogon citratus (DC.) Stapf et Lantana camara (Linn.) cultivées sur des sols ferrugineux tropicaux et vertiques. Thèse, Université Polytechnique de Bobo-Dioulasso (UPB). p.170

Senou I., Gnankambary Z., Some N. A., Sedogo P.M. 2014. Projection de trois espèces de plantes locales pour la phytoextraction de métaux lourds. Agronomie africaine, 26 (2), pp.155-166

Senou I., Gnankambary Z., Some N.A., Nacro H.B. 2018. Responses of five local plant species to metal exposure under controlled conditions. Int. J. Develop. Res. 8, pp. 18501-18506

Walkley A., Black J.A. 1934. An examination of the Degtjareff method for determining soil organic matter and a proposed modification of the chromatic acid titration method. Soil Science, 37 pp.29-38

Xiaomei L., Qitang W., Banks M.K. 2005. Effect of simultaneous establishment of Sedum alfredii and Zea mays on heavy metal accumulation in plants. International journal of Phytoremediation, 7 (1), pp. 43-53

Zorrig W. 2011. Recherche et caractérisation de déterminants contrôlant l'accumulation de cadmium chez la laitue " Lactuca sativa ".Thèse. Montpellier SupAgro, p. 250 